\title{
Analytic Tangent Irradiance Environment Maps for Anisotropic Surfaces
}

\author{
Soham Uday Mehta ${ }^{1}$, Ravi Ramamoorthi ${ }^{1}$, Mark Meyer ${ }^{2}$ and Christophe Hery ${ }^{2}$ \\ ${ }^{1}$ University of California, Berkeley $\quad{ }^{2}$ Pixar Animation Studios
}

\begin{abstract}
Environment-mapped rendering of Lambertian isotropic surfaces is common, and a popular technique is to use a quadratic spherical harmonic expansion. This compact irradiance map representation is widely adopted in interactive applications like video games. However, many materials are anisotropic, and shading is determined by the local tangent direction, rather than the surface normal. Even for visualization and illustration, it is increasingly common to define a tangent vector field, and use anisotropic shading. In this paper, we extend spherical harmonic irradiance maps to anisotropic surfaces, replacing Lambertian reflectance with the diffuse term of the popular Kajiya-Kay model. We show that there is a direct analogy, with the surface normal replaced by the tangent. Our main contribution is an analytic formula for the diffuse Kajiya-Kay BRDF in terms of spherical harmonics; this derivation is more complicated than for the standard diffuse lobe. We show that the terms decay even more rapidly than for Lambertian reflectance, going as $l^{-3}$, where $l$ is the spherical harmonic order, and with only 6 terms $(l=0$ and $l=2$ ) capturing $99.8 \%$ of the energy. Existing code for irradiance environment maps can be trivially adapted for real-time rendering with tangent irradiance maps. We also demonstrate an application to offline rendering of the diffuse component of fibers, using our formula as a control variate for Monte Carlo sampling.
\end{abstract}

Categories and Subject Descriptors (according to ACM CCS): I.3.3 [Computer Graphics]: Picture/Image Generation- I.3.7 [Computer Graphics]: Three-Dimensional Graphics and Realism—shading

\section{Introduction}

Realistic lighting is important for visual realism, and interactive applications often use environment maps. For Lambertian surfaces, a popular approach is to use low-order spherical harmonics to represent the irradiance map [RH01a], which is evaluated at run-time for a given surface normal at each pixel. These methods are based on an analytic formula for the spherical harmonic coefficients of the Lambertian BRDF [BJ01, RH01b], and it suffices to use terms up to order 2 , which are simply quadratic polynomials. Cast shadows and interreflection are not explicitly considered; however unlike more accurate and expensive precomputed relighting methods [SKS02, NRH03], no precomputation is required (except the usually minimal one-time effort of projecting an environment map into spherical harmonics). Storage requirements are also minimal. For these reasons, the method is widely adopted in real-time rendering-for example, in video games such as the Halo series [CL08].

However, some materials are anisotropic, with shading depending on the local tangent direction; in many cases, there is not even a well-defined surface normal. Common examples are hair and fur, where we store only the local fiber direction or tangent (many derivations consider a thin cylinder, but the geometry for that cylinder is not usually tessellated, just as standard BRDF models do not explicitly tessellate the micro-facets). We use the standard Kajiya-Kay reflectance model [KK89] developed for hair and fur. (The alternative anisotropic Ward model [War92] or alternatives like Ashikhmin-Shirley only include a Lambertian diffuse term). Anisotropy also affects many other surfaces, such as those with grooves or threads. In other cases, we may deliberately seek to shade opaque objects anisotropically for visualization [Ban94] (in fact, the Banks model is essentially the same as Kajiya-Kay). Recent work has shown that tangent fields can easily be created on general geometry, making it likely that tangent-space anisotropic shading will become widely used [FSDH07, CDS10].

Anisotropic reflections under point sources are common in computer graphics, and there is some early work on anisotropically filtering environment maps for specular re- 

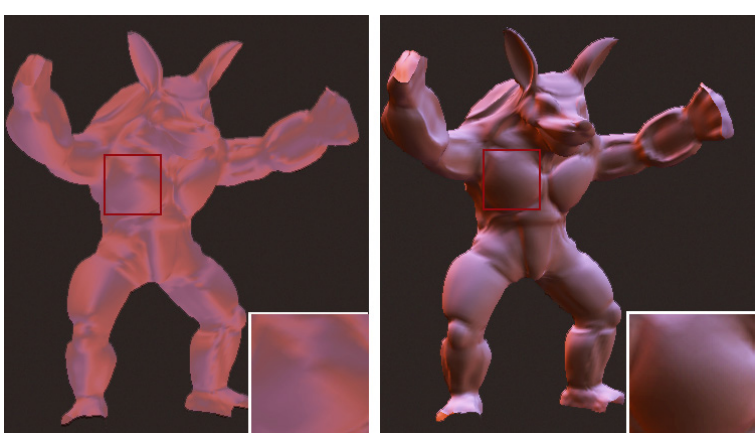

Figure 1: Comparison of environment-mapped armadillo rendering with tangents (left) and with standard Lambertian normals (right). Tangents are defined procedurally with longitude lines on a sphere, using the point with the same global surface normal. Notice the interesting shading effects on the left, that strongly suggest an anisotropic material.

flections [KVHS00]. However, there is no analogous technique to spherical harmonic irradiance maps, for rendering diffuse anisotropic surfaces in environment lighting, and these visual effects have therefore been largely absent in interactive (or even offline) computer graphics.

In this paper, we show how to create spherical harmonic tangent irradiance maps, where the normal is simply replaced by the tangent. As with the original work, we do not consider shadows and interreflections, and also do not require precomputation or per-vertex/pixel storage.

We focus on diffuse anisotropic reflections only, where a simple analytic formula can be derived, in contrast to most recent research that has studied specular reflections from fibers. This is similar to the separation in standard BRDF models of diffuse and specular terms. No simple expression or compact representation is available for environment mapping the specular term. However, numerical methods already exist for specular Kajiya-Kay [RH02] (that method could potentially also be extended to the diffuse term, although this is not demonstrated; in any case, our analytic formula makes the implementation much simpler). More recently, numerical approaches for rendering the recent specular Marschner model $\left[\mathrm{MJC}^{*} 03\right.$ ] with precomputation [RZL $\left.{ }^{*} 10\right]$ have been developed. These specular effects could trivially be added linearly to our method, but we do not include them in our renderings, to highlight the new visual effects enabled by environment-mapped diffuse anisotropic reflection.

Our main contribution is an analytic formula for the spherical harmonic coefficients in the diffuse term of the KajiyaKay BRDF. This derivation is considerably more involved than in the Lambertian case, and cannot be found in standard references. However, it leads to a more compact final formula. In fact, terms decay even more rapidly as $l^{-3}$, with all odd spherical harmonic orders $l$ vanishing (in contrast, the $l=1$ term contributes in the Lambertian case). Only 6 terms
( 1 constant term for $l=0$ and 5 quadratic terms for $l=2$ ) suffice to capture $99.8 \%$ of the energy with higher accuracy, compared to the 9 term Lambertian model. The result is still a quadratic polynomial, so existing code and shaders for irradiance environment maps can trivially be adapted to the anisotropic case. We demonstrate results with procedurally defined tangents (Fig. 1), tangents acquired from real data (Fig. 4), tangent fields created on geometric models (Fig. 5), and fur (Fig. 6) and cloth (Fig. 7). Our supplementary video shows real-time rendering of these effects. The formula can also be used as a control variate for Monte Carlo sampling of the diffuse component of hair fibers (Figs. 8, 9).

\section{Preliminaries}

The reflection equation for the irradiance map can be written as,

$$
E(\mathbf{t})=\int_{S^{2}} L(\boldsymbol{\omega}) f(\boldsymbol{\omega}, \mathbf{t}) d \boldsymbol{\omega},
$$

where the irradiance $E$ is expressed as a function of the tangent direction $\mathbf{t}$ (instead of the normal), and the integral is over the sphere of directions $S^{2} . L$ is the incident environment map, while $f$ is the net transfer function. The BRDF formula for the Kajiya-Kay diffuse term is [KK89],

$$
f(\boldsymbol{\omega}, \mathbf{t})=\sin (\boldsymbol{\omega}, \mathbf{t})=\sqrt{1-(\boldsymbol{\omega} \cdot \mathbf{t})^{2}},
$$

where we are considering the sine of the angle between light and tangent directions (instead of cosine for the surface normal), and we use the trigonometric relation for the sine, in terms of the cosine. Unlike Lambertian reflection, this formula is symmetric with respect to the tangent direction (there is no "back hemisphere.")

It is convenient to normalize the above equation,

$$
f(\boldsymbol{\omega} \cdot \mathbf{t})=\frac{\rho}{\pi^{2}} \sin \theta=\frac{\rho}{\pi^{2}} \sqrt{1-(\boldsymbol{\omega} \cdot \mathbf{t})^{2}} .
$$

We make explicit that $f$ depends only on $\boldsymbol{\omega} \cdot \mathbf{t}=\cos \theta$; this will be important for the spherical harmonic analysis. $\rho$ is the diffuse albedo in the range $[0 \ldots 1]$ and the factor of $1 / \pi^{2}$ ensures that $E(\mathbf{t})=1$ if $\rho=1$ and $L(\boldsymbol{\omega})=1$, i.e., energy is conserved in a uniform white dome. (To derive that result, consider the right-hand side of equation 1 , $\left(1 / \pi^{2}\right) \int_{\theta} \int_{\phi}(\sin \theta) \sin \theta d \theta d \phi=(2 / \pi) \int_{0}^{\pi} \sin ^{2} \theta d \theta$, and upon integrating, we get $(1 / \pi) \int_{0}^{\pi}(1-\cos 2 \theta) d \theta=1-0=1$.)

The $1 / \pi^{2}$ normalization should be compared to the $1 / \pi$ factor for Lambertian reflectance. (Note that [RH01a], among others do not include the $1 / \pi$ factor in the spherical harmonic coefficients, so that their constant coefficient is actually $\pi$ instead of 1 for Lambertian reflectance. In this paper, we find it more convenient to normalize the BRDF.) For the remainder of this paper, we set $\rho=1$, since it is simply a multiplier on the irradiance map (or more correctly, the shading is computed as $\rho E(\mathbf{t}))$. 


\section{Analytic Spherical Harmonic Formula}

Equation 1 can now be written,

$$
E(\mathbf{t})=\int_{S^{2}} L(\boldsymbol{\omega}) f(\boldsymbol{\omega} \cdot \mathbf{t}) d \boldsymbol{\omega} .
$$

This is a very similar form as for Lambertian reflectance, only with the normal replaced with the tangent, and with a different form for $f$. Hence, we can find a simple spherical harmonic formula. In particular, explanding the irradiance and lighting in spherical harmonics $Y_{l m}$ where $l$ is the major index $(l \geq 0)$, and $-l \leq m \leq+l$,

$$
\begin{aligned}
L(\boldsymbol{\omega}) & =\sum_{l=0}^{\infty} \sum_{m=-l}^{+l} L_{l m} Y_{l m}(\boldsymbol{\omega}) \\
E(\mathbf{t}) & =\sum_{l=0}^{\infty} \sum_{m=-l}^{+l} E_{l m} Y_{l m}(\mathbf{t}) .
\end{aligned}
$$

We also expand $f$ in terms of spherical harmonic coefficients $f_{l}$,

$$
f(\boldsymbol{\omega} \cdot \mathbf{t})=\frac{\sin \theta}{\pi^{2}}=\sum_{l=0}^{\infty} f_{l} Y_{l 0}(\theta) .
$$

Since $f$ depends only on $\boldsymbol{\omega} \cdot \mathbf{t}$ or on elevation angle $\theta$ alone, there is no azimuthal or $\phi$ dependence, and we can simply use the radially symmetric terms $Y_{l 0}$ (the coefficients for $Y_{l m}$ with $m \neq 0$ are 0 ).

It is known that equation 4 is a spherical convolution [BJ01, RH01a], with a simple product formula in the frequency domain,

$$
E_{l m}=\sqrt{\frac{4 \pi}{2 l+1}} f_{l} L_{l m}=A_{l} L_{l m}
$$

where we define $A_{l}=\sqrt{4 \pi /(2 l+1)} f_{l}$. Until this point, the derivation is very similar to the Lambertian case. However, the form of $f$ (and hence $A_{l}$ and $f_{l}$ ) is rather different, making the derivations more complicated. One of the main contributions of this paper is the derivation and analysis of these coefficients, which follows. However, readers more interested in practical implementation can skip ahead to Sec. 4.

\subsection{Spherical Harmonic Expansion of Kajiya-Kay}

The function $f(\theta)=\sin \theta$, which is different from the clamped cosine in the Lambertian case. This makes finding $f_{l}$ somewhat harder. Including the solid angle measure, we write,

$$
f_{l}=\frac{1}{\pi^{2}} \int_{\theta=0}^{\pi} \int_{\phi=0}^{2 \pi} \sin \theta Y_{l 0}(\theta) \sin \theta d \theta d \phi .
$$

The value for $A_{l}$ simply multiplies this by $\sqrt{4 \pi /(2 l+1)}$. We also know that $Y_{l 0}(\theta)=\sqrt{(2 l+1) /(4 \pi)} P_{l}(\cos \theta)$, where $P_{l}$ are the Legendre polynomials. Putting this together, and also doing the $\phi$ integral that has value $2 \pi$,

$$
A_{l}=\frac{2}{\pi} \int_{0}^{\pi} P_{l}(\cos \theta) \sin ^{2} \theta d \theta
$$

To get some intuition, consider the first three Legendre polynomials, $P_{0}(u)=1, P_{1}(u)=u, P_{2}(u)=\frac{1}{2}\left(3 u^{2}-1\right)$. One important property is that they are odd for odd $l$, and even for even $l$. Since $\sin ^{2} \theta$ is even, $A_{l}$ vanishes for odd $l$ (unlike in Lambertian reflection, even the $l=1$ term will vanish). Finally, we can simplify the above expression by using $\sin ^{2} \theta=\frac{1}{2}(1-\cos 2 \theta)$,

$$
A_{l}=\frac{1}{\pi} \int_{0}^{\pi} P_{l}(\cos \theta)(1-\cos 2 \theta) d \theta
$$

While this expression appears deceptively simple, an analytic formula seems beyond the capabilities of symbolic manipulation systems like Mathematica. To solve this integral, we start with a result for Legendre polynomials, given in [Mac67] (page 106). If $r$ and $s$ are integers, it is known that

$$
\int_{0}^{\pi} P_{r+2 s}(\cos \theta) \cos (r \theta) d \theta=\frac{\Gamma\left(s+\frac{1}{2}\right) \Gamma\left(r+s+\frac{1}{2}\right)}{\Gamma(s+1) \Gamma(r+s+1)},
$$

where $\Gamma$ is Euler's gamma function. In our case, since $A_{l}$ vanishes for odd $l$, we can compute the formulae for $A_{2 n}$ with $l=2 n$. The integrand in equation 10 is $(1-\cos 2 \theta)=$ $(\cos 0 \theta-\cos 2 \theta)$, so we set $r=0,2$. Comparing equations 10 and 11, we see that $(r+2 s)=2 n$, so that the two terms of the integrand correspond to $(r, s)=(0, n)$ and $(r, s)=(2, n-1)$. Therefore,

$$
A_{2 n}=\frac{1}{\pi}\left(\frac{\Gamma\left(n+\frac{1}{2}\right) \Gamma\left(n+\frac{1}{2}\right)}{\Gamma(n+1) \Gamma(n+1)}-\frac{\Gamma\left(n-\frac{1}{2}\right) \Gamma\left(n+\frac{3}{2}\right)}{\Gamma(n) \Gamma(n+2)}\right) .
$$

To simplify this expression, we would like the second term on the right-hand side to have the same denominator as the first. To bring it into the same form, we use the identity that $\Gamma(u+1)=u \Gamma(u)$,

$$
\begin{aligned}
\Gamma(n) \Gamma(n+2) & =\frac{\Gamma(n+1)}{n} \cdot(n+1) \Gamma(n+1) \\
& =\Gamma(n+1) \Gamma(n+1) \cdot \frac{n+1}{n} .
\end{aligned}
$$

The numerator of the second term can also be simplified,

$$
\begin{aligned}
\Gamma\left(n-\frac{1}{2}\right) \Gamma\left(n+\frac{3}{2}\right) & =\frac{\Gamma\left(n+\frac{1}{2}\right)}{n-\frac{1}{2}} \cdot\left(n+\frac{1}{2}\right) \Gamma\left(n+\frac{1}{2}\right) \\
& =\Gamma\left(n+\frac{1}{2}\right) \Gamma\left(n+\frac{1}{2}\right) \cdot \frac{2 n+1}{2 n-1} .
\end{aligned}
$$

The numerator and denominator for the two terms on the right-hand side in equation 12 can now be combined,

$$
A_{2 n}=\frac{1}{\pi}\left(\frac{\Gamma\left(n+\frac{1}{2}\right)}{\Gamma(n+1)}\right)^{2}\left(1-\frac{2 n+1}{2 n-1} \cdot \frac{n}{n+1}\right) .
$$

We can still simplify this expression considerably. First note that by definition $\Gamma(n+1)=n$ ! in the denominator, so we can simply use the factorial of $n$. For the numerator,

$$
\Gamma\left(n+\frac{1}{2}\right)=\Gamma\left(\frac{1}{2}\right) \cdot \frac{1}{2} \cdot \frac{3}{2} \ldots \frac{2 n-1}{2}=\frac{\sqrt{\pi}}{2^{n}} \cdot \frac{(2 n) !}{2^{n} \cdot n !} .
$$




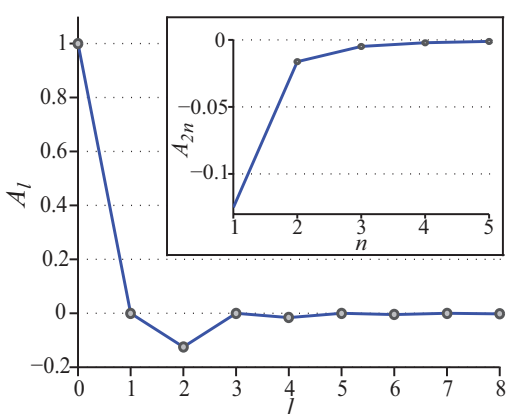

(a)

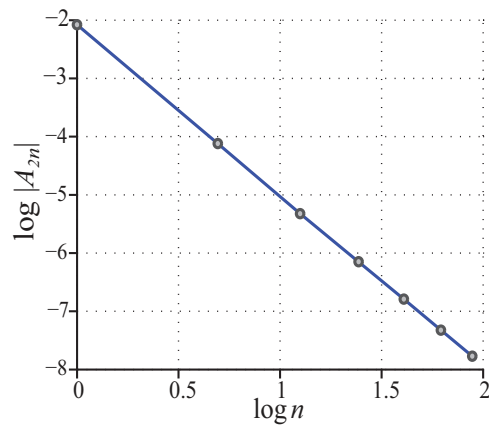

(b)

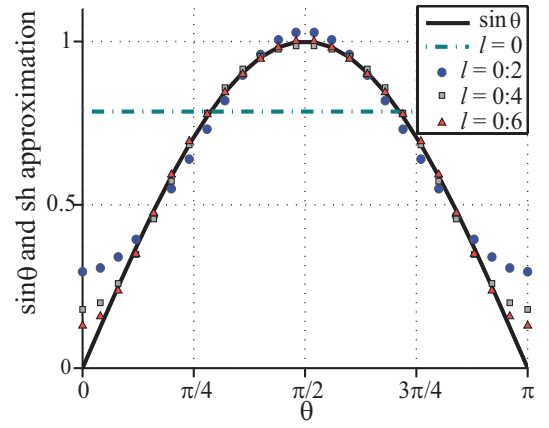

(c)

Figure 2: (a) Plots of the spherical harmonic coefficients $A_{l}$ for the Kajiya Kay diffuse term. Note that the odd terms vanish, and all even terms except the first are negative. (b) The coefficient magnitude falls off very rapidly, decaying as $l^{-3}$, as seen in this loglog plot. (c) The original Kajiya-Kay BRDF function or sine kernel, and its approximation for increasing harmonic orders. Constant and quadratic terms $l=0,2$ together capture $99.8 \%$ of the BRDF energy.

Finally, the fractional term on the right of equation 15 can be expanded and simplifed down to $(-1) /[(n+1)(2 n-1)]$. Putting this all together, we arrive at our final formula,

$$
A_{2 n}=\frac{-1}{(n+1)(2 n-1)}\left(\frac{(2 n) !}{\left(2^{n} \cdot n !\right)^{2}}\right)^{2} .
$$

We have also verified this formula for small and large values of $n$ against Mathematica, both analytically and numerically.

\subsection{Analysis and Discussion}

We can simply plug increasing values of $n$ into equation 17 to obtain numerical values for the first few terms,

$$
\begin{aligned}
A_{0} & =1 \\
A_{2} & =-\frac{1}{8}=-0.125 \\
A_{4} & =-\frac{1}{64}=-0.01625 \\
A_{6} & =-\frac{5}{1024} \approx-0.00488281 \\
A_{8} & =-\frac{35}{16384} \approx-0.00213623 \\
A_{10} & =-\frac{147}{131072} \approx-0.00112521 .
\end{aligned}
$$

Note that all coefficients except the first are negative, unlike the alternating signs for Lambertian.

It is also instructive to consider the asymptotic falloff of the coefficients with $n$. The fractional part in equation 17 clearly decays quadratically as $n^{-2}$. For the factorials, it is easiest to use Stirling's approximation in equation 15 , with $\log \Gamma(u) \approx\left(u-\frac{1}{2}\right) \log u$,

$\log \left(\frac{\Gamma\left(n+\frac{1}{2}\right)}{\Gamma(n+1)}\right)^{2}=2\left(\log \Gamma\left(n+\frac{1}{2}\right)-\log \Gamma(n+1)\right) \approx-\log n$,

which means the factorial term asymptotically drops off as $n^{-1}$. Therefore, the entire expression for $A_{2 n}$ falls off as $n^{-3}$, which is even somewhat faster than for Lambertian reflectance (where the falloff is as $n^{-5 / 2}$ ). This is not really surprising, since tangent shading is a smooth function without even the clamping of the cosine term for the Lambertian case. In Fig. 2(a,b), we plot the values of $A_{2 n}$ on a linear and logarithmic scale. The logarithmic plot clearly shows the asymptotic $n^{-3}$ falloff, and the linear plot clearly shows the very rapid decay of values (which also vanish for odd $A_{l}$ ). Figure 2(c) also shows increasingly accurate approximations to the sine kernel with higher orders. Note that the approximations in Fig. 2(c) are always positive (unlike in the Lambertian case, where the spherical harmonic approximation to the clamped cosine can be slightly negative and therefore unphysical in the back hemisphere).

We can also consider what fraction of the energy of the BRDF is captured by a certain number of harmonic terms. For this, it is more conventional to use $f_{l}=\sqrt{(2 l+1) /(4 \pi)} A_{l}$. By Parseval's theorem, the total (squared) energy is that in the original BRDF, with $f^{2}$ written as $f^{2}(\theta)=\pi^{-4} \sin ^{2} \theta$ which is given by $8 /\left(3 \pi^{3}\right)$,

$\iint\left(\pi^{-4} \sin ^{2} \theta\right) \sin \theta d \theta d \phi=2 \pi^{-3} \int_{0}^{\pi} \sin ^{3} \theta d \theta=\frac{4}{3} \cdot 2 \pi^{-3}$.

The first coefficient $\left(f_{0}\right)^{2}=1 /(4 \pi)$ already captures $92.5 \%$ of the energy (compared with $37.5 \%$ for the Lambertian case). For anisotropic surfaces, there is no contribution from the first order mode $l=1$ (which accounts for $50 \%$ of the energy in the Lambertian case). The quadratic $l=2$ mode has total energy $\left(f_{2}\right)^{2}=5 /(256 \pi)$ and accounts for $7.2 \%$ of the total energy.

Together, orders $l=0$ as well as $l=2$ account for approximately $99.8 \%$ of the total energy in the BRDF. Therefore, the quadratic polynomial or order 2 spherical harmonic approximation is even more accurate than in the Lambertian 
S. U. Mehta, R. Ramamoorthi, M. Meyer and C. Hery / Analytic Tangent Irradiance Environment Maps for Anisotropic Surfaces

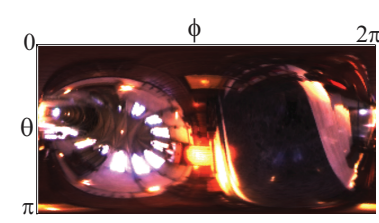

(a) Environment map

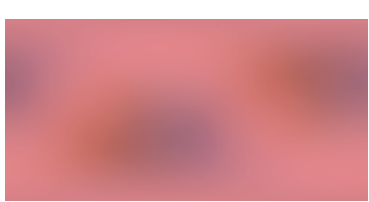

(b) Ground Truth Tangent Irrad.

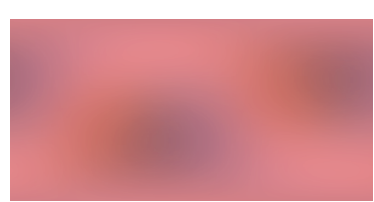

(c) Spherical Harmonics order 2

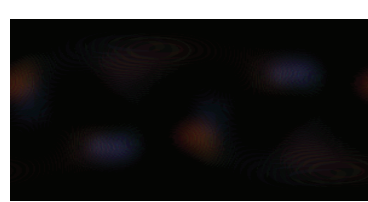

(d) Error

Figure 3: Accuracy of the 6 term spherical harmonic approximation: (a) The enivronment map on a $(\theta, \phi)$ grid, (b) Ground truth brute force angular domain integration for the irradiance, $(c)$ Rendering with the quadratic (six term) spherical harmonic approximation and $(d)$ the per-pixel error between the six term approximation and the brute force integration, magnified 5 times. We see that the quadratic approximation is essentially indistinguishable from ground truth.

case. Moreover, since the linear term with $l=1$ is missing, we need only compute 6 spherical harmonic terms (the single constant term for $(l, m)=(0,0)$ and the 5 order 2 modes with $l=2$, where $-2 \leq m \leq 2$ ). In Fig. 3, we show the high accuracy of the 6 term approximation over the entire tangent irradiance map.

\section{Implementation}

In this section, we give the precise formulae to actually implement spherical harmonic tangent irradiance maps. We assume the spherical harmonic lighting coefficients $L_{00}$ and $L_{2 m}$ have been computed and are available, as for Lambertian irradiance maps. Expanding equation 7 explicitly for a given tangent direction,

$$
E(\mathbf{t}) \approx A_{0} L_{00} Y_{00}+A_{2} \sum_{m=-2}^{2} L_{2 m} Y_{2 m}(\mathbf{t}) .
$$

To proceed further, we tabulate the real forms of the relevant spherical harmonics (we find it convenient to write them as quadratic polynomials of the cartesian components of $\mathbf{t}$ ),

$$
\begin{aligned}
Y_{00} & =\sqrt{\frac{1}{4 \pi}} \\
\left(Y_{2-2} ; Y_{2-1} ; Y_{21}\right) & =\sqrt{\frac{15}{4 \pi}}(x y ; y z ; x z) \\
Y_{20} & =\sqrt{\frac{5}{16 \pi}}\left(3 z^{2}-1\right) \\
Y_{22} & =\sqrt{\frac{15}{16 \pi}}\left(x^{2}-y^{2}\right) .
\end{aligned}
$$

Plugging equations 18 and 21 into equation 20, and taking numerical values (with $(x, y, z)$ being the cartesian components of the unit tangent vector $\mathbf{t})$,

$$
\begin{aligned}
E(\mathbf{t}) & =b_{0} L_{00}+2 b_{1}\left(L_{2-2} x y+L_{2-1} y z+L_{21} x z\right) \\
& +b_{1} L_{22}\left(x^{2}-y^{2}\right)+b_{2} L_{20} z^{2}+b_{3} L_{20} .
\end{aligned}
$$

$$
\begin{array}{lll}
b_{0}= & \sqrt{\frac{1}{4 \pi}} & \approx+0.282095 \\
b_{1}= & -\frac{1}{16} \sqrt{\frac{15}{4 \pi}}=-\frac{1}{8} \sqrt{\frac{15}{16 \pi}} & \approx-0.0682843 \\
b_{2}= & -\frac{3}{8} \sqrt{\frac{5}{16 \pi}} & \approx-0.118272 \\
b_{3}= & \frac{1}{8} \sqrt{\frac{5}{16 \pi}} & \approx+0.0394239 .
\end{array}
$$

It is also possible to write this with a quadratic matrix formula,

$$
E(\mathbf{t})=\mathbf{t} \cdot M \mathbf{t},
$$

where $\mathbf{t}$ is augmented to have a constant term (i.e., a homogeneous 4-vector common in graphics with $\mathbf{t}=(x, y, z, 1))$, and the symmetric matrix $4 \times 4$ matrix $M$ is given by

$$
M=\left(\begin{array}{cccc}
b_{1} L_{22} & b_{1} L_{2-2} & b_{1} L_{21} & 0 \\
b_{1} L_{2-2} & -b_{1} L_{22} & b_{1} L_{2-1} & 0 \\
b_{1} L_{21} & b_{1} L_{2-1} & b_{2} L_{20} & 0 \\
0 & 0 & 0 & b_{0} L_{00}+b_{3} L_{20}
\end{array}\right)
$$

All of these equations are straightforward and can be implemented in a few lines of shader code for real-time rendering. Moreover, they have essentially the same numerical form as the shaders for the Lambertian case, which makes adapting those shaders to tangent irradiance maps straightforward. We have implemented all of our results with simple GLSL shaders.

\section{Results}

We demonstrate a variety of results, using spherical harmonic tangent irradiance maps, and many different ways of specifying the tangents. Our supplemental video shows real-time rendering of many of these examples. These results bring a new visual capability into computer graphics, since environment-mapped diffuse anisotropic reflection has been largely absent in previous work.

First, Fig. 1 shows the characteristic visual patterns obtained by tangent shading, as opposed to isotropic Lambertian reflectance. To define the tangents in this example, we used the global surface normal to map a given pixel to a point 
S. U. Mehta, R. Ramamoorthi, M. Meyer and C. Hery / Analytic Tangent Irradiance Environment Maps for Anisotropic Surfaces

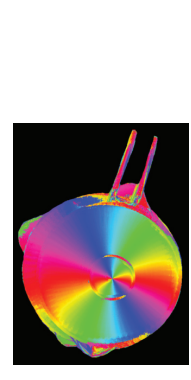

(a)

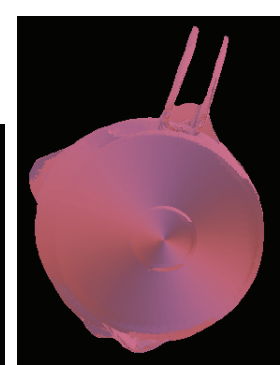

(b)

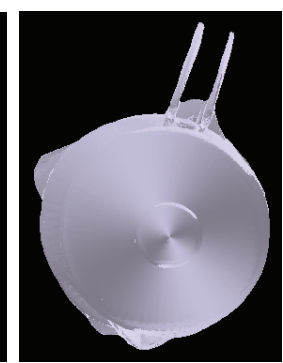

(c)

Figure 4: Tangent irradiance maps, using tangent data from a real dataset: (a): A visualization of the tangent fields (hue corresponds to polar angle in the plane of the figure; tangents lie in concentric circles for most of this object). (b) and (c): Images under Grace Cathedral and Uffizi environment maps respectively. Note how the image is approximately constant along radial lines from the center, since those pixels have similar tangents.

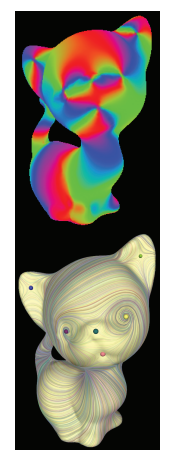

(a)

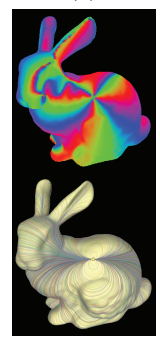

(d)

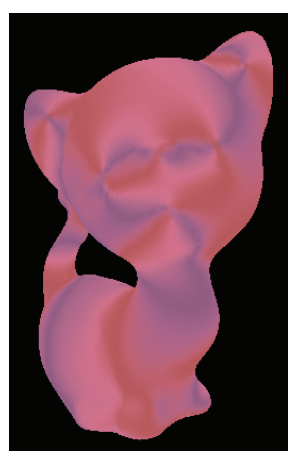

(b)

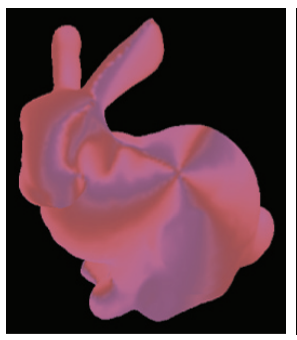

(e)

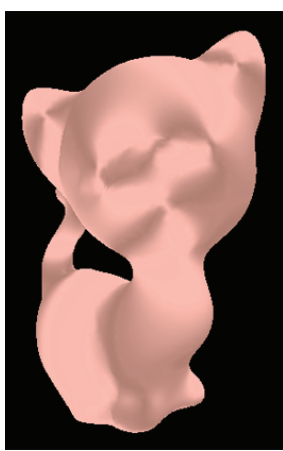

(c)

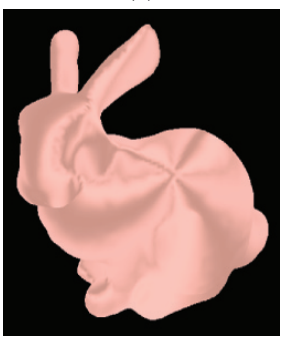

(f)

Figure 5: Diffuse anisotropic shading to render objects with a user-specified tangent field. On the left $(a, d)$, we show the tangent field using both the hue angle in Fig. 4 and the flow line visualization of [CDS2010]. Rendered images use Grace Cathedral (b,e) and Galileo's Tomb (c,f) environment maps, and show characteristic anisotropic patterns, which conform to the flow and singularities of the tangent field. These real-time diffuse anisotropic environment-mapped images present a new visual capability.

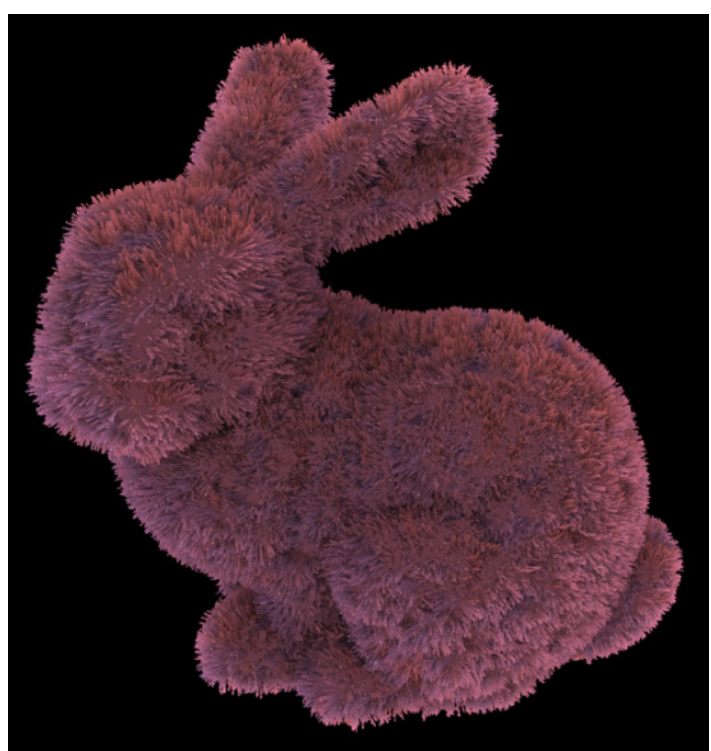

Figure 6: A dense mesh of a furry bunny with ambient occlusion and spherical harmonic tangent irradiance maps.

on the unit sphere, with tangents defined procedurally along the corresponding longitude lines.

In Fig. 4, we apply our method to tangents obtained by acquiring a real object [HLHZ08] (note that we use only the tangent fields, not any other property of the measured reflectance). This allows us to visualize the object with realistic tangents and diffuse anisotropic shading, in an environment map.

Figure 5 shows our method applied to synthetic objects, with a user-specified tangent field (from [CDS10]). Designing tangent vector fields is becoming more common, and our method allows for more interesting visualizations of these objects in complex lighting, enabling novel types of visual depictions.

Figure 6 applies spherical harmonic tangent irradiance maps to the fur on a more complex million-polygon bunny model with tangents along the hair direction, showing that the method is also relevant to hair or fur. For this example, we also compute ambient occlusion at each vertex, which simply multiplies the tangent shading. While shadows and global illumination are not explicitly considered in our formula, this example shows that we can easily include standard real-time approximations like ambient occlusion. Figure 7 shows another example, a scarf model with 1.5 million triangles. The tangents are defined along the direction of the fibers, and we combine spherical harmonic tangent irradiance maps with precomputed ambient occlusion.

While we have focused mostly on real-time applications, we can also use our analytic formula as a control variate for Monte Carlo sampling (we thank Simon Premoze for first 
S. U. Mehta, R. Ramamoorthi, M. Meyer and C. Hery / Analytic Tangent Irradiance Environment Maps for Anisotropic Surfaces

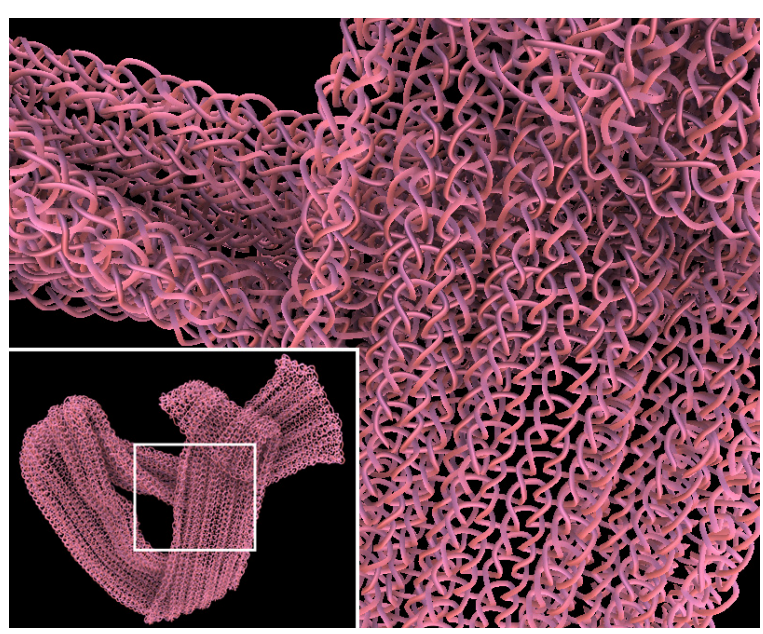

Figure 7: Scarf (1.5 million triangles) with vertex tangents defined along the direction of the fiber containing the vertex.

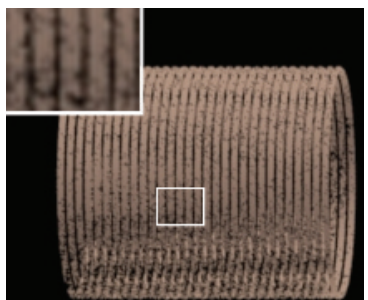

(a) Control Variates, 1 Sample

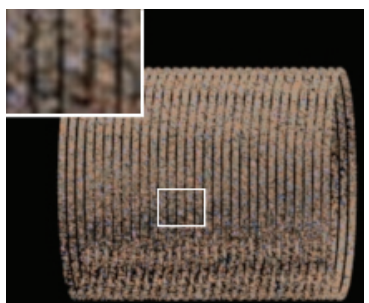

(b) No Cont. Variates 1 sample
Figure 8: Comparison of diffuse hair-like fibers rendered (a) with and $(b)$ without control variates. The cylinder is composed of 30 hair fibers, shaped like circles and assembled next to each other. We use a very low sample count of only 1 sample per pixel to demonstrate the key effects, and to make the noise more apparent when printing or viewing. The control variate result in (a) has considerably less noise than (b).

suggesting this use of control variates, in the context of Lambertian shading). Control variates are an important tool for variance reduction in Monte Carlo, and have been used before for direct lighting [CAM08].

Introducing visibility $V(\boldsymbol{\omega})$ into equation 1 and defining the complement or shadowing as $\bar{V}=1-V$,

$$
\int_{S^{2}} L(\boldsymbol{\omega}) V(\boldsymbol{\omega}) f(\boldsymbol{\omega}, \mathbf{t}) d \boldsymbol{\omega}=E(\mathbf{t})-\int_{S^{2}} L(\boldsymbol{\omega}) \bar{V}(\boldsymbol{\omega}) f(\boldsymbol{\omega}, \mathbf{t}) d \boldsymbol{\omega} .
$$

In this case, $E(\mathbf{t})$ (which assumes $V=1$ since we do not consider cast shadows) is the control variate, and can be analytically computed using our formula. The remaining expression is sampled in the standard way with Monte Carlo. If most points are actually visible, the sampled integral on the right-hand side will vanish $(\bar{V}=0)$, and we will get a result which is more accurate and has less noise than direct
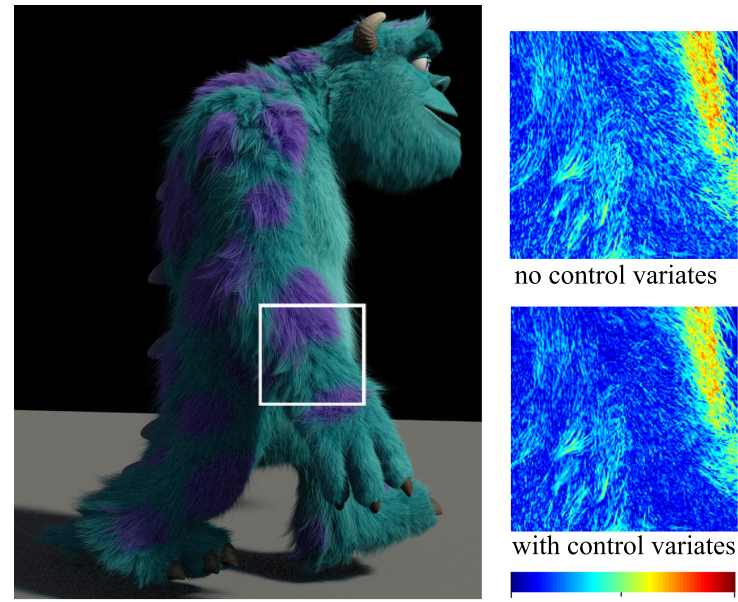

no control variates

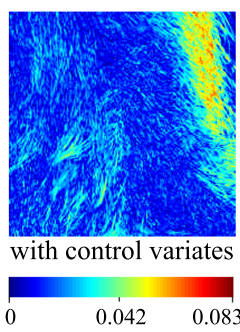

Rendered using control variates, 12 samples

$0.042 \quad 0.083$

Figure 9: The spherical harmonic control variate sampling method used in production rendering of fur. We also show false-color (cold to warm) closeups of the absolute error versus ground truth; control variates have lower error. Image copyright (2012) Pixar. All Rights Reserved.

Monte Carlo evaluation. In practice, we slightly modify the above expression to also be consistent in shadowed regions, by blending between the original expression and the control variate method. In particular, we compute the image $B$,

$$
\begin{aligned}
B & =(1-\alpha)\left(\int_{S^{2}} L(\boldsymbol{\omega}) V(\boldsymbol{\omega}) f(\boldsymbol{\omega}, \mathbf{t}) d \boldsymbol{\omega}\right) \\
& +\alpha\left(E(\mathbf{t})-\int_{S^{2}} L(\boldsymbol{\omega}) \bar{V}(\boldsymbol{\omega}) f(\boldsymbol{\omega}, \mathbf{t}) d \boldsymbol{\omega}\right),
\end{aligned}
$$

where $\alpha$ is the fraction of samples that are visible (when this is close to 1 , we use the control variate result in equation 25 , but when it is close to 0 , the unshadowed control variate is much less useful). The same lighting samples are used for both integrals above, so there is only one sampling pass and minimal overhead.

We apply the method to rendering the diffuse component of hair-like fibers with importance sampling of the lighting in Fig. 8 (the specular component would be added separately with BRDF importance sampling). Using control variates substantially reduces noise. Figure 9 shows the method applied to production rendering, where the fur uses the diffuse Kajiya-Kay BRDF. The insets show closeups of the absolute difference with respect to ground truth, and it is clear that the control variate technique has lower error.

\section{Conclusions and Future Work}

In this paper, we have developed a simple method for rendering the diffuse component of anisotropic surfaces under environment maps. Our method is based on a new analytic formula for the spherical harmonic coefficients of the 
Kajiya-Kay model and uses only a 6 term spherical harmonic representation of the tangent irradiance map. It is trivial to adapt existing Lambertian spherical harmonic shading methods to tangent shading, and we demonstrate a number of examples. One limitation, shared with Lambertian irradiance maps, is that we do not consider cast shadows and interreflections; this could be more significant in our case since the sine kernel includes lighting from both hemispheres. We believe this is a reasonable approximation, given that we introduce a visual effect that has rarely been seen before, especially in interactive computer graphics. It should also be possible to combine our approach with ambient occlusion, as in Figs. 6 and 7, or with spherical harmonic visibility by first multiplying the lighting and visibility as in [SKS02], and then directly applying our method to the properly shadowed lighting. We discuss this further in the appendix. We believe that anisotropic materials and tangent-space visualization are growing in importance, and have taken an important step to allow environment mapping of these surfaces.

Acknowledgements: We thank Milos Hasan for helpful discussions and for providing models for many of the scenes in the paper. We also thank Keenan Crane and Michael Holroyd for datasets.

\section{References}

[Ban94] BANKS D.: Illumination in diverse codimensions. In SIGGRAPH 94 (1994), pp. 327-334. 1

[BJ01] BASRI R., JACOBS D.: Lambertian Reflectance and Linear Subspaces. In International Conference on Computer Vision (2001), pp. 383-390. 1, 3

[CAM08] Clarberg P., AKenine-Moller T.: Exploiting visibility correlation in direct illumination. Computer Graphics Forum (EGSR 08) 27, 4 (2008), 1125-1136. 7

[CDS10] Crane K., Desbrun M., Schroder P.: Trivial connections on discrete surfaces. Computer Graphics Forum (SGP 2010) 29, 5 (2010), 1525-1533. 1, 6

[CL08] CHEN H., LIU X.: Lighting and materials of Halo 3. http://developer.amd.com/gpu_assets/S2008-ChenLighting_and_Material_of_Halo3.pdf, 2008. 1

[FSDH07] Fisher M., Schroder P., Desbrun M., Hoppe H.: Design of tangent vector fields. ACM Transactions on Graphics (SIGGRAPH 07) 26, 3 (2007). 1

[HLHZ08] Holroyd M., LAwrence J., Humphreys G., ZICKLER T.: A photometric approach for estimating normals and tangents. ACM Transactions on Graphics (SIGGRAPH ASIA 08) 27,5 (2008), 133:1-133:9. 6

[KK89] KAJIYA J., KAY T.: Rendering Fur with Three Dimensional Textures. In SIGGRAPH 89 (1989), pp. 271-280. 1, 2

[KVHS00] Kautz J., Vázquez P., Heidrich W., Seidel H.: A Unified Approach to Prefiltered Environment Maps. In EuroGraphics Rendering Workshop 00 (2000), pp. 185-196. 2

[Mac67] MACROBERT T.: Spherical harmonics: an elementary treatise on harmonic functions with applications. Dover Publications, 1967. 3

[MJC*03] Marschner S., Jensen H., Cammarano M., WORLEY S., HANRAHAN P.: Light scattering from human hair fibers. ACM Transactions on Graphics (SIGGRAPH 03) 22, 3 (2003), 780-791. 2
[NRH03] Ng R., RAMAMOORThi R., HanRahan P.: AllFrequency Shadows using Non-Linear Wavelet Lighting Approximation. ACM Transactions on Graphics (SIGGRAPH 03) 22, 3 (2003), 376-381. 1

[RH01a] RAmamoorthi R., HANRAHAN P.: An Efficient Representation for Irradiance Environment Maps. In SIGGRAPH 01 (2001), pp. 497-500. 1, 2, 3, 8

[RH01b] RAMAMOORTHI R., HANRAHAN P.: On the relationship between Radiance and Irradiance: Determining the illumination from images of a convex Lambertian object. JOSA A 18, 10 (2001), 2448-2459. 1

[RH02] Ramamoorthi R., HanRahan P.: Frequency Space Environment Map Rendering. ACM Transactions on Graphics (SIGGRAPH 02) 21, 3 (2002), 517-526. 2

[RZL*10] Ren Z., Zhou K., Li T., HuA W., Guo B.: Interactive hair rendering under environment lighting. ACM Transactions on Graphics (SIGGRAPH 10) 29, 4 (2010). 2

[SKS02] Sloan P., Kautz J., Snyder J.: Precomputed Radiance Transfer for Real-Time Rendering in Dynamic, LowFrequency Lighting Environments. ACM Transactions on Graphics (SIGGRAPH 02) 21, 3 (2002), 527-536. 1, 8

[War92] WARD G. J.: Measuring and Modeling Anisotropic Reflection. In Computer Graphics (SIGGRAPH 92) (1992), pp. 265-272. 1

\section{Appendix: Multiplying by Visibility (Future Work)}

We could directly use the shadowed lighting given by $L^{\prime}(\boldsymbol{\omega})=$ $L(\boldsymbol{\omega}) V(\boldsymbol{\omega})$ in our method,

$$
L_{l m}^{\prime}=\sum_{p=0}^{\infty} \sum_{q=-p}^{p} \sum_{r=0}^{\infty} \sum_{s=-r}^{r} C_{l m ; p q, r s} L_{p q} V_{r s},
$$

where $C_{l m ; p q, r s}$ are the Clebsch-Gordan coefficients.

An interesting special case is when we want to combine with surface shading, such as the Lambertian term,

$$
\begin{aligned}
V(\boldsymbol{\omega}) & =g(\boldsymbol{\omega} \cdot \mathbf{n})=\sum_{r=0}^{\infty} \sum_{s=-r}^{r} H_{r} Y_{r s}(\boldsymbol{\omega}) Y_{r s}(\mathbf{n}) \\
V_{r s} & =H_{r} Y_{r s}(\mathbf{n}),
\end{aligned}
$$

where $H_{r}$ are normalized spherical harmonic coefficients for the function $g(\boldsymbol{\omega} \cdot \mathbf{n})$ given by $H_{r}=\sqrt{4 \pi /(2 r+1)} g_{r}$. If we wanted to modulate with Lambertian shading, the coefficients $H_{r}$ are given by [RH01a], and we can plug $V_{r s}$ into equation 27 . If we only wanted to restrict to the upper hemisphere ( $g$ is a Heaviside step function), coefficients fall off even faster (exponential decay),

$$
\begin{aligned}
H_{r} & =\sqrt{\frac{4 \pi}{2 r+1}} \int_{\theta=0}^{\pi / 2} \int_{\phi=0}^{2 \pi} Y_{r 0}(\theta) \sin \theta d \theta d \phi=2 \pi \int_{0}^{1} P_{r}(u) d u \\
& =2 \pi \frac{\sqrt{\pi}}{2 \Gamma\left(1-\frac{r}{2}\right) \Gamma\left(\frac{3+r}{2}\right)},
\end{aligned}
$$

where it is understood that $H_{r}=0$ when $r>0$ is even. The first few terms are $H_{0}=2 \pi, H_{1}=\pi, H_{2}=0, H_{3}=-\pi / 4, H_{4}=0$. Plugging into equation 27 and setting all the summation limits, and noting that $q=m-s$ (for real harmonics $q= \pm m \pm s$ ), we obtain a simple formula,

$$
L_{l m}^{\prime} \approx \sum_{r=0}^{1} \sum_{s=-r}^{r} \sum_{p=l-r}^{l+r} C_{l m ; p(m-s), r s} H_{r} L_{p, m-s} Y_{r s}(\mathbf{n}) .
$$

